\title{
On Moment Generating Function of Generalized Order Statistics From Extended Type II Generalized Logistic Distribution
}

\author{
Devendra Kumar \\ Department of Statistics, Amity Institute of Applied Sciences \\ Amity University, Noida-201 303, India \\ E-mail: devendrastats@gmail.com \\ Received 29 November 2013 \\ Accepted 17 April 2014
}

\begin{abstract}
In this paper, explicit expressions and some recurrence relations are derived for marginal and joint moment generating functions of generalized order statistics from extended type II loglogistic distribution. Further the results are deduced for moments of $k$-th record values and ordinary order statistics.
\end{abstract}

Key words Generalized order statistics; order statistics; record values; marginal and joint moment generating function; recurrence relations ; extended type II loglogistic distribution.

AMS Subject Classification: $62 \mathrm{G} 30,62 \mathrm{E} 10$.

\section{Introduction}

A random variable $X$ is said to have extended type II generalized logistic distribution if its probability density function $(p d f)$ is of the form

$$
f(x)=\frac{\alpha e^{-\alpha x}}{\left(1+e^{-x}\right)^{\alpha+1}}, \quad-\infty<x<\infty, \alpha>0
$$


and the corresponding survival function is

$$
\bar{F}(x)=\frac{e^{-\alpha x}}{\left(1+e^{-x}\right)^{\alpha}}, \quad-\infty<x<\infty, \alpha>0
$$

where

$$
\bar{F}(x)=1-F(x) .
$$

For more details on this distribution and its application one may refer to Balakrishnan and Leung [4].

The logistic distribution plays an important role in growth curve have made it one of the many important statistical distributions. The shape of the logistic distribution that is similar to that of the normal distribution makes it simpler and also profitable on suitable occasions to replace the normal by the logistic distribution with negligible errors in the respective theories. Kamps [6] introduced and extensively studied the generalized order statistics (gos). The order statistics, sequential order statistics, Stigler's order statistics, record values are special cases of gos. Suppose $X(1, n, m, k), \ldots, X(n, n, m, k)$ are $n$ gos from an absolutely continuous distribution function $(d f) F(x)$ with the corresponding $p d f f(x)$. Their joint $p d f$ is

$$
k\left(\prod_{j=1}^{n-1} \gamma_{j}\right)\left(\prod_{i=1}^{n-1}\left[1-F\left(x_{i}\right)\right]^{m} f\left(x_{i}\right)\right)\left[1-F\left(x_{n}\right)\right]^{k-1} f\left(x_{n}\right)
$$

for $F^{-1}(0+)<x_{1} \leq x_{2} \leq \ldots \leq x_{n}>F^{-1}(1), m \geq-1, \gamma_{r}=k+(n-r)(m+1)>0$, $r=1,2, \ldots, n-1, k \geq 1$ and $n$ is a positive integer.

Choosing the parameters appropriately, models such as ordinary order statistics $\left(\gamma_{i}=n-i+1 ; i=1,2, \ldots, n\right.$, i.e. $\left.m_{1}=m_{2}=\ldots=m_{n-1}=0, k=1\right), k-$ th record values $\left(\gamma_{i}=k\right.$ i.e. $\left.m_{1}=m_{2}=\ldots=m_{n-1}=-1, \quad k \in N\right)$, sequential order statistics $\left(\gamma_{i}=(n-i+1) \alpha_{i}\right.$; $\left.\alpha_{1}, \alpha_{2}, \ldots, \alpha_{n}>0\right)$, order st atistics with non-integral sample size $\left(\gamma_{i}=\alpha-i+1 ; \alpha>0\right)$, Pfeifer's record values $\left(\gamma_{i}=\beta_{i} ; \beta_{1}, \beta_{2}, \ldots, \beta_{n}>0\right)$ and progressive type II censored order statistics $\left(m_{i} \in N, k \in N\right)$ are obtained (Kamps [6], Kamps and Cramer [7]).

The marginal $p d f$ of $r$-th $g o s, X(r, n, m, k)$, is 


$$
f_{X(r, n, m, k)}(x)=\frac{C_{r-1}}{(r-1) !}[\bar{F}(x)]^{\gamma_{r}-1} f(x) g_{m}^{r-1}(F(x))
$$

and the joint pdf of $X(r, n, m, k)$ and $X(s, n, m, k), 1 \leq r<s \leq n$, is

$$
\begin{aligned}
f_{X(r, n, m, k), X(s, n, m, k)}(x, y) & =\frac{C_{s-1}}{(r-1) !(s-r-1) !}[\bar{F}(x)]^{m} f(x) g_{m}^{r-1}(F(x)) \\
\times & {\left[h_{m}(F(y))-h_{m}(F(x))\right]^{s-r-1}[\bar{F}(y)]^{\gamma_{s}-1} f(y), \quad x<y, }
\end{aligned}
$$

where

$$
\begin{aligned}
& C_{r-1}=\prod_{i=1}^{r} \gamma_{i}, \\
& h_{m}(x)= \begin{cases}-\frac{1}{m+1}(1-x)^{m+1} & , m \neq-1 \\
-\log (1-x) & , m=-1\end{cases}
\end{aligned}
$$

and

$$
g_{m}(x)=h_{m}(x)-h_{m}(0), \quad x \in[0,1) .
$$

Recurrence relations are interesting in their own right. They are useful in reducing the number of operations necessary to obtain a general form for the function under consideration. Furthermore, they are used in characterizing distributions, which in important area, permitting the identification of population distribution from the properties of the sample.

Ahsanullah and Raq ab [1], Raqab an d Ahsanullah [14, 15] have established recurrence relations for moment generating functions of record values from Pareto and Gumble, power function and extreme value distributions.

Recurrence relations for marginal and joint moment generating functions of gos from power function distribution and Erlang-truncated exponential distribution are derived by Saran and Pandey [16] and Kulshrestha et al. [8] respectively. Saran and Pandey [17] and Kumar [10, $11,12]$ have established recurrence relations for marginal and join t moment generating functions of lower generalized order statistics from power function, generalized logistic, Marshall-Olkin extended logistic and extended type I g eneralized logistic distribution respectively. Al-Hussaini et al. [2, 3] have established recurrence relations for conditional and joint moment generating functions of gos based on mixed population, respectively. Kumar [9] have established explicit expressi ons and some recurrence relations for moment generating function of record values from generalized logistic. 
In the present study, we establish exact expressions and some recurrence relations for marginal and joint moment generating functions of gos from extended type II generalized logistic distribution. Results for order statistics and record values are deduced as special cases.

\section{Relations for marginal moment generating function}

Note that for extended type II generalized logistic distribution defined in (1.1)

$$
\alpha \bar{F}(x)=\left(1+e^{-x}\right) f(x) .
$$

The relation in (2.1) will be exploited in this paper to derive exact expressions and some recurrence relations for the moment generating function of gos from the extended type II generalized logistic distribution.

Let us denote the marginal moment generating functions of $X(r, n, m, k)$ by $M_{X(r, n, m, k)}(t)$ and its $j$-th derivative by $M_{X(r, n, m, k)}^{(j)}(t)$.

We shall first establish some basic results which may be helpful in proving the main result.

Lemma 2.1: For the extended type II generalized logistic distribution as given in (1.1) and any non-negative and finite integers $a$ and $b$

$$
I(a, 0)=\alpha B(\alpha(a+1)-t, t+1)
$$

where

$$
I(a, b)=\int_{-\infty}^{\infty} e^{t x}[\bar{F}(x)]^{a} f(x) g_{m}^{b}(F(x)) d x .
$$

Proof: From (2.3), we have

$$
I(a, 0)=\int_{-\infty}^{\infty} e^{t x}[\bar{F}(x)]^{a} f(x) d x .
$$

Making the substitution $z=[\bar{F}(x)]^{1 / \alpha},(2.4)$ reduces to

$$
I(a, 0)=\alpha \int_{0}^{1}(1-z)^{t} z^{\alpha(a+1)-t-1} d z
$$

and hence the result given in (2.2).

Lemma 2.2: Let $I(a, b)$ be as $g$ iven in (2.3), where $a \geq 0$ and $b \geq 0$ are integers. If $m \neq-1$

$$
I(a, b)=\frac{1}{(m+1)^{b}} \sum_{u=0}^{b}(-1)^{u}\left(\begin{array}{l}
b \\
u
\end{array}\right) I(a+u(m+1), 0)
$$




$$
=\frac{\alpha}{(m+1)^{b}} \sum_{u=0}^{b}(-1)^{u}\left(\begin{array}{l}
b \\
u
\end{array}\right) B(\alpha(a+(m+1) u+1)-t, t+1), t \neq 0,
$$

if $m=-1$

$$
I(a, b)=(-1)^{b} \alpha^{b+1} \frac{\partial^{b}}{\partial v^{b}} B(v, t+1),
$$

where $v=\alpha(a+1)-t>0$ and $B(a, b), a, b>0$ is the beta function.

Proof: On expanding $g_{m}^{b}(F(x))=\left[\frac{1}{m+1}\left\{1-(\bar{F}(x))^{m+1}\right\}\right]^{b}$ binomially in $(2.3)$, we get when $m \neq-1$

$$
\begin{aligned}
I(a, b) & =\frac{1}{(m+1)^{b}} \sum_{u=0}^{b}(-1)^{u}\left(\begin{array}{l}
b \\
u
\end{array}\right) \int_{-\infty}^{\infty} e^{t x}[\bar{F}(x)]^{a+u(m+1)} f(x) d x \\
& =\frac{1}{(m+1)^{b}} \sum_{u=0}^{b}(-1)^{u}\left(\begin{array}{l}
b \\
u
\end{array}\right) I(a+u(m+1), 0) .
\end{aligned}
$$

Making use of Lemma 2.1, we establish the result given in (2.6).

When $m=-1$ we have that

$$
I(a, b)=\int_{-\infty}^{\infty} e^{t x}[\bar{F}(x)]^{a} f(x)[-\log \bar{F}(x)]^{b} d x .
$$

By substitution $z=[\bar{F}(x)]^{1 / \alpha},(2.8)$, we get

$$
\begin{aligned}
I(a, b) & =\alpha^{b+1} \int_{0}^{1}(1-z)^{t} z^{\alpha(a+1)-t-1}[-\log z]^{b} d z \\
& =(-1)^{b} \alpha^{b+1} \int_{0}^{1}(1-z)^{t} z^{\alpha(a+1)-t-1}[\log z]^{b} d z .
\end{aligned}
$$

Using the following results
a) $\int_{0}^{p}\left(p^{\delta}-x^{\delta}\right)^{\beta-1} x^{\alpha-1}[\log x]^{n} d x=\frac{p^{\delta(\beta-1)}}{\delta} \frac{\partial^{n}}{\partial \alpha^{n}}\left[p^{\alpha} B\left(\beta, \frac{\alpha}{\delta}\right)\right], \quad p, \delta, \alpha, \beta>0$
b) $\frac{\partial^{r} B(a, b)}{\partial b^{r}}=\sum_{k=0}^{r-1}\left(\begin{array}{c}r-1 \\ k\end{array}\right)\left[\psi^{(r-k+1)}(b)-\psi^{(r-k-1)}(a+b)\right] \frac{\partial^{k} B(a, b)}{\partial b^{k}}$, 
where $B(a, b), a, b>0$ is the beta fun ction $\psi^{(k)}(x)$ is the $k-$ th derivative of $\psi(x)=\frac{d \log \Gamma(x)}{d x}=\frac{\Gamma^{\prime}(x)}{\Gamma(x)}, \quad x \neq 0,-1,-2, \ldots$ which is a digamma function. The result given in (2.7) is proved, in view of result (a).

Theorem 2.1: For extended type II generalized logistic distribution as given in (1.1) and for $1 \leq r \leq n, k=1,2, \ldots, m \neq-1$

$$
\begin{aligned}
& M_{X(r, n, m, k)}(t)=\frac{C_{r-1}}{(r-1) !} I\left(\gamma_{r}-1, r-1\right) \\
& =\frac{\alpha C_{r-1}}{(r-1) !(m+1)^{r-1}} \sum_{u=0}^{r-1}(-1)^{u}\left(\begin{array}{c}
r-1 \\
u
\end{array}\right) B\left(\alpha \gamma_{r-u}, t+1\right),
\end{aligned}
$$

if $m=-1$

$$
\begin{aligned}
M_{X(r, n,-, k)}(t) & =\frac{k^{r}}{(r-1) !} I(k-1, r-1) \\
= & (\alpha k)^{r}(-1)^{r-1} \frac{\partial^{r-1}}{\partial(\alpha k-t)^{r-1}} B(\alpha k-t, t+1),
\end{aligned}
$$

where $I\left(\gamma_{r}-1, r-1\right), I(k-1, r-1)$ are as defined in (2.3). Using the result (b) recursively, we can obtain the moments of any value of $r$.

Proof: From (1.4), we have

$$
M_{X(r, n, m, k)}(t)=\frac{C_{r-1}}{(r-1) !} \int_{-\infty}^{\infty} e^{t x}[\bar{F}(x)]^{\gamma_{r}-1} f(x) g_{m}^{r-1}(F(x)) d x
$$

and hence the result given in (2.12). Making use of (2.6) in (2.9), we establish the result given in (2.10).

When $m=-1$, we have that

$$
M_{X(r, n,-1, k)}(t)=\frac{k^{r}}{(r-1) !} \int_{-\infty}^{\infty} e^{t x}[\bar{F}(x)]^{k-1} f(x)[-\log (\bar{F}(x))]^{r-1} d x
$$

and hence the result given in (2.11). Making use of (2.7) in (2.11), we establish the result given 


\section{Special cases}

i) Putting $m=0, k=1$ in (2.10), the ex plicit formula for marginal moment generating function of order statistics from the extended type II generalized logistic distribution can be obtained as

$$
M_{X_{r: n}}(t)=\alpha C_{r: n} \sum_{u=0}^{r-1}(-1)^{u}\left(\begin{array}{c}
r-1 \\
u
\end{array}\right) B(\alpha(n-r+1+u), t+1),
$$

where

$$
C_{r: n}=\frac{n !}{(r-1) !(n-r) !} .
$$

ii) Setting $k=1$ in (2.12), we get the explicit expression for marginal moment generating function of upper $k$ record values from extended type II generalized logistic distribution can be obtained as

$$
M_{X(r, n,-1,1)}(t)=M_{X_{U(r)}}(t)=\alpha^{r}(-1)^{r-1} \frac{\partial^{r-1}}{\partial(\alpha-t)^{r-1}} B(\alpha-t, t+1), \quad t \neq 0 .
$$

A recurrence relation for marginal moment generating function for gos from $d f$ (1.2) can be obtained in the following theorem.

Theorem 2.2: For the distribution given in (1.1) and for $2 \leq r \leq n, n \geq 2$ and $k=1,2 \ldots$

$$
\begin{array}{r}
\left(1-\frac{t}{\alpha \gamma_{r}}\right) M_{X(r, n, m, k)}^{(j)}(t)=M_{X(r-1, n, m, k)}^{(j)}(t)+\frac{j}{\alpha \gamma_{r}} M_{X(r, n, m, k)}^{(j-1)}(t) \\
+\frac{1}{\alpha \gamma_{r}}\left\{t M_{X(r, n, m, k)}^{(j)}(t-1)+j M_{X(r, n, m, k)}^{(j-1)}(t-1)\right\}
\end{array}
$$

Proof: From (1.4), we have

$$
M_{X(r, n, m, k)}(t)=\frac{C_{r-1}}{(r-1) !} \int_{-\infty}^{\infty} e^{t x}[\bar{F}(x)]^{\gamma_{r}-1} f(x) g_{m}^{r-1}(F(x)) d x
$$

Integrating by parts treating $[\bar{F}(x)]^{\gamma_{r}-1} f(x)$ for integration and rest of the integrand for differentiation, we get

$$
M_{X(r, n, m, k)}(t)=M_{X(r-1, n, m, k)}(t)+\frac{t C_{r-1}}{\gamma_{r}(r-1) !} \int_{-\infty}^{\infty} e^{t x}[\bar{F}(x)]^{\gamma_{r}} g_{m}^{r-1}(F(x)) d x
$$

the constant of integration vanishes since the integral considered in (2.14) is a definite integral. On using (2.1), we obtain 


$$
\begin{aligned}
M_{X(r, n, m, k)}(t)= & M_{X(r-1, n, m, k)}(t)+\frac{t C_{r-1}}{\alpha \gamma_{r}(r-1) !}\left\{\int_{-\infty}^{\infty} e^{t x}[\bar{F}(x)]^{\gamma_{r}-1} f(x) g_{m}^{r-1}(F(x)) d x\right. \\
& \left.+\int_{-\infty}^{\infty} e^{(t-1) x}[\bar{F}(x)]^{\gamma_{r}-1} f(x) g_{m}^{r-1}(F(x)) d x\right\} \\
& =M_{X(r-1, n, m, k)}(t)+\frac{t}{\alpha \gamma_{r}}\left\{M_{X(r, n, m, k)}(t)+M_{X(r, n, m, k)}(t-1)\right\}
\end{aligned}
$$

Differentiating both the sides of (2.15) $j$ times with respect to $t$, we get

$$
\begin{aligned}
M_{X(r, n, m, k)}^{(j)}(t) & =M_{X(r-1, n, m, k)}^{(j)}(t)+\frac{t}{\alpha \gamma_{r}} M_{X(r, n, m, k)}^{(j)}(t) \\
& +\frac{j}{\alpha \gamma_{r}} M_{X(r, n, m, k)}^{(j-1)}(t)+\frac{t}{\alpha \gamma_{r}} M_{X(r, n, m, k)}^{(j)}(t-1)+\frac{j}{\alpha \gamma_{r}} M_{X(r, n, m, k)}^{(j-1)}(t-1) .
\end{aligned}
$$

The recurrence relation in equation (2.13) is derived simply by rewriting the above equation. By differentiating both sides of equation (2.13) with respect to $t$ and then setting $t=0$, we obtain the recurrence relations for moments of gos from extended type II generalized logistic distribution in the form

$$
\begin{aligned}
E\left[X^{j}(r, n, m, k)\right] & =E\left[X^{j}(r-1, n, m, k)\right] \\
+\frac{j}{\alpha \gamma_{r}} & \left\{E\left[X^{j-1}(r, n, m, k)\right]+E[\phi(X(r, n, m, k))]\right\}
\end{aligned}
$$

where

$$
\phi(x)=x^{j-1} e^{-x}
$$

Remark 2.1: Putting $m=0, k=1$ in (2.13) and (2.16), we can get the relations for marginal moment generating function of order statistics for extended ty pe II g eneralized logistic distribution in the form

$$
\begin{array}{r}
\left(1-\frac{t}{\alpha(n-r+1)}\right) M_{X_{r: n}}^{(j)}(t)=M_{X_{r-1: n}}^{(j)}(t)+\frac{j}{\alpha(n-r+1)} M_{X_{r: n}}^{(j-1)}(t) \\
+\frac{1}{\alpha(n-r+1)}\left\{t M_{X_{r: n}}^{(j)}(t-1)+j M_{X_{r: n}}^{(j-1)}(t-1)\right\}
\end{array}
$$

and

$$
E\left[X_{r: n}^{j}\right]=E\left[X_{r-1: n}^{j}\right]+\frac{j}{\alpha(n-r+1)}\left\{E\left[X_{r: n}^{j-1}\right]+E\left[\phi\left(X_{r: n}\right)\right]\right\}
$$


Remark 2.2: Setting $m=-1$ and $k \geq 1$, in (2.13) and (2.16), relations for record values can be obtained as

$$
\begin{aligned}
\left(1-\frac{t}{\alpha k}\right) M_{Z_{r}^{(k)}}^{(j)}(t)= & M_{Z_{r-1}^{(k)}}^{(j)}(t)+\frac{j}{\alpha k} M_{Z_{r}^{(k)}}^{(j-1)}(t) \\
& +\frac{1}{\alpha k}\left\{t M_{Z_{r}^{(k)}}^{(j)}(t-1)+j M_{Z_{r}^{(k)}}^{(j-1)}(t-1)\right\}
\end{aligned}
$$

and

$$
E\left[\left(Z_{r}^{(k)}\right)^{j}\right]=E\left[\left(Z_{r-1}^{(k)}\right)^{j}\right]+\frac{j}{\alpha k}\left\{E\left[\left(Z_{r}^{(k)}\right)^{j-1}\right]+E\left[\phi\left(Z_{r: n}\right)\right]\right\}
$$

for $k=1$

$$
E\left[X_{U(r)}^{j}\right]=E\left[X_{U(r-1)}^{j}\right]+\frac{j}{\alpha}\left\{E\left[X_{U(r)}^{j-1}\right]+E\left[\phi\left(X_{U(r)}\right)\right]\right\} .
$$

\section{Relations for joint moment generating function}

Before coming to the main results we shall prove the following Lemmas.

Lemma 3.1 For the extended type II generalized logistic distribution as given in (1.1) and non-negative integers $a, b$ and $c$

$$
I(a, 0, c)=\alpha \sum_{p=0}^{\infty} \frac{(1-v)_{(p)}}{p !\left[c+1+\left(p-t_{2}\right) / \alpha\right]} B\left(\alpha(a+c+2)+p-t_{1}-t_{2}, t_{1}+1\right),
$$

where

$$
I(a, b, c)=\int_{-\infty}^{\infty} \int_{x}^{\infty} e^{t_{1} x+t_{2} y}[\bar{F}(x)]^{a} f(x)\left[h_{m}(F(y))-h_{m}(F(x))\right]^{b}[\bar{F}(y)]^{c} f(y) d y d x .
$$

Proof: From (3.2), we have

$$
\begin{aligned}
I(a, 0, c) & =\int_{-\infty}^{\infty} \int_{x}^{\infty} e^{t_{1} x+t_{2} y}[\bar{F}(x)]^{a} f(x)[\bar{F}(y)]^{c} f(y) d y d x \\
& =\int_{-\infty}^{\infty} e^{t_{1} x}[\bar{F}(x)]^{a} f(x) G(x) d x,
\end{aligned}
$$

where

$$
G(x)=\int_{x}^{\infty} e^{t_{2} y}[\bar{F}(y)]^{c} f(y) d y .
$$

By setting $z=[\bar{F}(y)]^{1 / \alpha}$ in (3.4) we get

$$
\begin{aligned}
& G(x)=\alpha \int_{0}^{[\bar{F}(x)]^{1 / \alpha}}(1-z)^{t_{2}} z^{\alpha(c+1)-t_{2}-1} d z \quad \text { (See Pearson [13]). } \\
&=\sum_{p=0}^{\infty} \frac{(1-v)_{(p)}[\bar{F}(x)]^{c+1+\left(p-t_{2}\right) / \alpha}}{p !\left[c+1+\left(p-t_{2}\right) / \alpha\right]}, \quad v=t_{2}+1 \quad \\
& \text { Published by Atlantis Press } \\
& \text { Copyright: the authors }
\end{aligned}
$$


On substituting the above expression of $G(x)$ in (3.3), we find that

$$
I(a, 0, c)=\sum_{p=0}^{\infty} \frac{(1-v)_{(p)}}{p !\left[c+1+\left(p-t_{2}\right) / \alpha\right]} \int_{-\infty}^{\infty} e^{t_{1} x}[\bar{F}(x)]^{a+c+1+\left(p-t_{2}\right) / \alpha} f(x) d x .
$$

Again by setting $w=[\bar{F}(x)]^{1 / \alpha}$ in (3.5) and simplifying the resulting expression, we derive the relation given in (3.1).

Lemma 3.2: Let $I(a, b, c)$ be as given in (3.2) where $a \geq 0, b \geq 0$ and $c \geq 0$ are integers. If $m \neq-1$

$$
\begin{aligned}
I(a, b, c)= & \frac{1}{(m+1)^{b}} \sum_{v=0}^{b}(-1)^{v}\left(\begin{array}{l}
b \\
v
\end{array}\right) I(a+(b-v)(m+1), 0, c+v(m+1)) \\
& =\frac{\alpha}{(m+1)^{b}} \sum_{p=0}^{\infty} \sum_{v=0}^{b}(-1)^{v}\left(\begin{array}{l}
b \\
v
\end{array}\right) \frac{(1-v)_{(p)}}{p !\left[c+v(m+1)+1+\left(p-t_{2}\right) / \alpha\right]} \\
& \times B\left(\alpha(a+c+(m+1) b+2)+p-t_{1}-t_{2}, t_{1}+1\right)
\end{aligned}
$$

if $m=-1$

$$
I(a, b, c)=\sum_{p=0}^{\infty} \sum_{q=0}^{\infty} \sum_{u=0}^{b}(-1)^{a}\left(\begin{array}{l}
b \\
u
\end{array}\right) \frac{\alpha^{a+b+1} a_{p}(u)(1-\phi)(q)}{\left[c+1+\left(q-t_{2}\right) / \alpha\right]} \frac{\partial^{a+b-u}}{\partial v^{a+b-u}} B(v, t+1),
$$

where $v=\alpha(c+1)+q-t_{2}-t_{1}$ and $\phi=p+u+t_{2}+1$.

Proof: When $m \neq-1$, we have

$$
\begin{aligned}
{\left[h_{m}(F(y))-\right.} & \left.h_{m}(F(x))\right]^{b}=\frac{1}{(m+1)^{b}}\left[(\bar{F}(x))^{m+1}-(\bar{F}(y))^{m+1}\right]^{b} \\
& =\frac{1}{(m+1)^{b}} \sum_{v=0}^{b}(-1)^{v}\left(\begin{array}{l}
b \\
v
\end{array}\right)[\bar{F}(y)]^{v(m+1)}[\bar{F}(x)]^{(b-v)(m+1)} .
\end{aligned}
$$

Now substituting for $\left[h_{m}(F(y))-h_{m}(F(x))\right]^{b}$ in equation (3.2), we get

$$
I(a, b, c)=\frac{1}{(m+1)^{b}} \sum_{v=0}^{b}(-1)^{v}\left(\begin{array}{l}
b \\
v
\end{array}\right) I(a+(b-v)(m+1), 0, c+v(m+1)) .
$$

Making use of the Lemma 3.1, we established the result given in (3.7).

When $m=-1$, we have

$$
I(a, b, c)=\int_{-\infty}^{\infty} e^{t_{1} x}[-\log \bar{F}(x)]^{a} \frac{f(x)}{\bar{F}(x)} I(x) d x
$$


where

$$
\begin{aligned}
I(x)= & \int_{x}^{\infty} e^{t_{2} y}[\log (\bar{F}(x))-\log (\bar{F}(y))]^{b}[\bar{F}(y)]^{c} f(y) d y \\
& =\sum_{u=0}^{b}\left(\begin{array}{l}
b \\
u
\end{array}\right)[\log (\bar{F}(x))]^{b-u} \int_{x}^{\infty} e^{t_{2} y}[-\log (\bar{F}(y))]^{u}[\bar{F}(y)]^{c} f(y) d y,
\end{aligned}
$$

by setting $w=[\bar{F}(x)]^{1 / \alpha}$ we get

$$
I(x)=\alpha^{u+1} \sum_{u=0}^{b}\left(\begin{array}{l}
b \\
u
\end{array}\right)[\log (\bar{F}(x))]^{b-u} \int_{X}^{[\bar{F}(x)]^{1 / \alpha}}(1-z)^{t_{2}}[-\log z]^{u} z^{\alpha(c+1)-t_{2}-1} d z
$$

On using the logarithmic expansion

$$
[-\log (1-t)]^{i}=\left(\sum_{p=1}^{\infty} \frac{t^{p}}{p}\right)^{i}=\sum_{p=0}^{\infty} \alpha_{p}(i) t^{i+p},|t|<1,
$$

where $\alpha_{p}(i)$ is the coe fficient of $t^{i+p}$ in the expansion of $\left(\sum_{p=1}^{\infty} \frac{t^{p}}{p}\right)^{i}$ (Balakrishnan and

Cohen [5], Shawky and Bakoban [18]), integrating the resulting expression we get

$$
I(x)=\alpha^{u} \sum_{p=0}^{\infty} \sum_{q=0}^{\infty} \sum_{u=0}^{b}\left(\begin{array}{l}
b \\
u
\end{array}\right) a_{p}(u) \frac{(1-\phi)_{(q)}[\bar{F}(x)]^{c+1+\left(q-t_{2}\right) / \alpha}}{q !\left[c+1+\left(q-t_{2}\right) / \alpha\right]}[\log \bar{F}(x)]^{b-u} .
$$

On substituting the above expression of $I(x)$ in (3.9), we find that

$$
\begin{aligned}
I(a, b, c)= & \alpha^{u} \sum_{p=0}^{\infty} \sum_{q=0}^{\infty} \sum_{u=0}^{b}\left(\begin{array}{l}
b \\
u
\end{array}\right) a_{p}(u) \frac{(1-\phi)_{(q)}}{q !\left[c+1+\left(q-t_{2}\right) / \alpha\right]} \\
& \times \int_{-\infty}^{\infty} e^{t_{1} x}[-\log \bar{F}(x)]^{a+b-u}[\bar{F}(x)]^{c+\left(\left(q-t_{2}\right) / \alpha\right)-1} f(x) d x
\end{aligned}
$$

Again by setting $w=[\bar{F}(x)]^{1 / \alpha}$ in (3.10) and simplifying the resulting expression, we derive the relation given in (3.8).

Theorem 3.1 For extended type II generalized logistic distribution as given in (1.1) and for $1 \leq r<s \leq n, k=1,2, \ldots$ If $m \neq-1$,

$$
\begin{gathered}
M_{X(r, n, m, k), X(s, n, m, k)}\left(t_{1}, t_{2}\right)=\frac{C_{s-1}}{(r-1) !(s-r-1) !(m+1)^{r-1}} \sum_{u=0}^{r-1}(-1)^{u}\left(\begin{array}{c}
r-1 \\
u
\end{array}\right) \\
\times I\left(m+(m+1) u, s-r-1, \gamma_{s}-1\right) \\
=\frac{\alpha C_{s-1}}{(r-1) !(s-r-1) !(m+1)^{s-2}} \sum_{p=0}^{\infty} \sum_{q=0}^{\infty} \sum_{u=0}^{r-1 s-r-1} \sum_{v=0}(-1)^{u+v}\left(\begin{array}{c}
r-1 \\
u
\end{array}\right)\left(\begin{array}{c}
s-r-1 \\
v
\end{array}\right) \\
\times \frac{(1-v)(p)}{p !\left[\gamma_{s-v}+\left(p-t_{2}\right) / \alpha\right]} B\left(\alpha \gamma_{r-u}+p-t_{1}-t_{2}, t_{1}+1\right),
\end{gathered}
$$


if $m=-1$

$$
\begin{aligned}
M_{X(r, n,-1, k), X(s, n,-1, k)}\left(t_{1}, t_{2}\right)= & \frac{k^{s}}{(r-1) !(s-r-1) !} I(r-1, s-r-1, k-1) \\
= & \frac{(\alpha k)^{s}}{(r-1) !(s-r-1) !} \sum_{p=0}^{\infty} \sum_{q=0}^{\infty} \sum_{u=0}^{s-r-1}(-1)^{r-1}\left(\begin{array}{c}
s-r-1 \\
u
\end{array}\right) \frac{a_{p}(u)(1-\phi)_{(q)}}{\left[\alpha k+q-t_{2}\right]} \\
& \times \frac{\partial^{s-2-u}}{\partial v^{s-2-u}} B\left(\alpha k+q-t_{2}-t_{1}, t_{1}+1\right] .
\end{aligned}
$$

Proof From (1.3), we have

$$
\begin{gathered}
M_{X(r, n, m, k), X(s, n, m, k)}\left(t_{1}, t_{2}\right)=\frac{C_{s-1}}{(r-1) !(s-r-1) !} \int_{-\infty}^{\infty} \int_{X}^{\infty} e^{t_{1} x+t_{2} y}[\bar{F}(x)]^{m} f(x) \\
\times g_{m}^{r-1}(F(x))\left[h_{m}(F(y))-h_{m}(F(x))\right]^{s-r-1}[\bar{F}(y)]^{\gamma_{s}-1} f(y) d y d x .
\end{gathered}
$$

On expanding $g_{m}^{r-1}(F(x))$ binomially in (3.15) and simplifying the resulting expression, we have the result given in (3.11). Making use of (3.7) in (3.11), we establish the relation given in (3.12).

When $m=-1$, we have that

$$
\begin{gathered}
M_{X(r, n,-1, k), X(s, n,-1, k)}\left(t_{1}, t_{2}\right)=\frac{k^{s}}{(r-1) !(s-r-1) !} \int_{-\infty}^{\infty} \int_{X}^{\infty} e^{t_{1} x+t_{2} y}[-\log \bar{F}(x)]^{r-1} \\
\times[\log (\bar{F}(x))-\log (\bar{F}(x))]^{s-r-1}[\bar{F}(y)]^{k-1} \frac{f(x)}{\bar{F}(x)} f(y) d y d x
\end{gathered}
$$

Making use of (3.8) in (3.13), we establish the relation given in (3.14).

\section{Special cases}

i) Putting $m=0, k=1$ in (3.12), the explicit formula for joint moment generating function of order statistics for the extended type II generalized logistic distribution can be obtained as

$$
\begin{aligned}
& M_{X_{r: n} X_{s: n}}\left(t_{1}, t_{2}\right)=\alpha C_{r, s: n} \sum_{p=0}^{\infty} \sum_{q=0}^{\infty} \sum_{u=0}^{r-1} \sum_{v=0}^{s-r-1}(-1)^{u+v}\left(\begin{array}{c}
r-1 \\
u
\end{array}\right)\left(\begin{array}{c}
s-r-1 \\
v
\end{array}\right) \\
& \times \frac{(1-v)_{(p)}}{p !\left[(n-s+1+v)+\left(p-t_{2}\right) / \alpha\right]} B\left(\alpha(n-r+1+u)+p-t_{1}-t_{2}, t_{1}+1\right),
\end{aligned}
$$

where

$$
C_{r, s: n}=\frac{n !}{(r-1) !(s-r-1) !(n-s) !}
$$


ii) Setting $k=1$ in (3.14), we ded uce the explicit expression for joint moment generating function of upper record value for extended type II generalized logistic distribution (3.8) in the form

$$
\begin{aligned}
M_{X_{U(r)}, X_{U(s)}\left(t_{1}, t_{2}\right)} & =\frac{\alpha^{s}}{(r-1) !(s-r-1) !} \sum_{p=0}^{\infty} \sum_{q=0}^{\infty} \sum_{u=0}^{s-r-1}(-1)^{r-1}\left(\begin{array}{c}
s-r-1 \\
u
\end{array}\right) \\
& \times \frac{a_{p}(u)(1-\phi)_{(q)}}{\left[\alpha+q-t_{2}\right]} \frac{\partial^{s-2-u}}{\partial v^{s-2-u}} B\left(\alpha+q-t_{2}-t_{1}, t_{1}+1\right) .
\end{aligned}
$$

Making use of (2.1), we can derive the recurrence relations for joint moment generating function of gos from (1.5).

Theorem 3.2: For the $\mathrm{d}$ istribution given in (1.1) and for $1 \leq r<s \leq n, n \geq 2$ and $k=1,2, \ldots$

$$
\begin{aligned}
\left(1-\frac{t_{2}}{\alpha \gamma_{s}}\right) & M_{X(r, n, m, k), X(s, n, m, k)}^{(i, j)}\left(t_{1}, t_{2}\right)=M_{X(r, n, m, k), X(s-1, n, m, k)}^{(i, j)}\left(t_{1}, t_{2}\right) \\
& +\frac{j}{\alpha \gamma_{S}} M_{X(r, n, m, k), X(s, n, m, k)}^{(i, j)-1)}\left(t_{1}, t_{2}\right)+\frac{1}{\alpha \gamma_{s}}\left\{t_{2} M_{X(r, n, m, k), X(s, n, m, k)}^{(i, j)}\left(t_{1}, t_{2}-1\right)\right. \\
& \left.+j M_{X(r, n, m, k), X(s, n, m, k)}^{(i, j-1)}\left(t_{1}, t_{2}-1\right)\right\}
\end{aligned}
$$

Proof: Using (1.5), the joint moment generating function of $X(r, n, m, k)$ and $X(s, n, m, k)$ is given by

$$
\begin{aligned}
& M_{X(r, n, m, k), X(s, n, m, k)}\left(t_{1}, t_{2}\right) \\
& =\frac{C_{s-1}}{(r-1) !(s-r-1) !} \int_{-\infty}^{\infty}[\bar{F}(x)]^{m} f(x) g_{m}^{r-1}(F(x)) I(x) d x,
\end{aligned}
$$

where

$$
I(x)=\int_{X}^{\infty} e^{t_{1} x+t_{2} y}\left[h_{m}(F(y))-h_{m}(F(x))\right]^{s-r-1}[\bar{F}(y)]^{\gamma_{s}-1} f(y) d y .
$$

Solving the integral in $I(x)$ by parts and substituting the resulting expression in (3.17), we get

$$
\begin{aligned}
M_{X(r, n, m, k), X(s, n, m, k)}\left(t_{1}, t_{2}\right)=M_{X(r, n, m, k), X(s-1, n, m, k)}\left(t_{1}, t_{2}\right) \\
+\frac{t_{2} C_{s-1}}{\gamma_{s}(r-1) !(s-r-1) !} \int_{-\infty}^{\infty} \int_{X}^{\infty} e^{t_{1} X+t_{2} y}[\bar{F}(x)]^{m} f(x) g_{m}^{r-1}(F(x)) \\
\times\left[h_{m}(F(y))-h_{m}(F(x))\right]^{s-r-1}[\bar{F}(y)]^{\gamma_{s}} d y d x,
\end{aligned}
$$


the constant of in tegration vanishes since the integral in $I(x)$ is a definite integral. On using the relation (2.1), we obtain

$$
\begin{aligned}
& M_{X(r, n, m, k), X(s, n, m, k)}\left(t_{1}, t_{2}\right)=M_{X(r, n, m, k), X(s-1, n, m, k)}\left(t_{1}, t_{2}\right) \\
& \quad+\frac{t_{2}}{\alpha \gamma_{s}}\left\{M_{X(r, n, m, k), X(s, n, m, k)}\left(t_{1}, t_{2}\right)+M_{X(r, n, m, k), X(s, n, m, k)}\left(t_{1}, t_{2}-1\right)\right\}
\end{aligned}
$$

Differentiating both the sides of (3.18) $i$ times with respect to $t_{1}$ and then $j$ times with respect to $t_{2}$, we get

$$
\begin{aligned}
& M_{X(r, n, m, k), X(s, n, m, k)}^{(i, j)}\left(t_{1}, t_{2}\right)=M_{X(r, n, m, k), X(s-1, n, m, k)}^{(i, j)}\left(t_{1}, t_{2}\right) \\
& \quad+\frac{t_{2}}{\alpha \gamma_{s}} M_{X(r, n, m, k), X(s, n, m, k)}^{(i, j)}\left(t_{1}, t_{2}\right)+\frac{j}{\alpha \gamma_{s}} M_{X(r, n, m, k), X(s, n, m, k)}^{(i, j-1)}\left(t_{1}, t_{2}\right) \\
& \quad+\frac{t_{2}}{\alpha \gamma_{s}} M_{X(r, n, m, k), X(s, n, m, k)}^{(i, j)}\left(t_{1}, t_{2}-1\right)+\frac{j}{\alpha \gamma_{s}} M_{X(r, n, m, k), X(s, n, m, k)}^{(i, j-1)}\left(t_{1}, t_{2}-1\right),
\end{aligned}
$$

which, when rewritten gives the recurrence relation in (3.16).

One can also note that Theorem 2.1 can be deduced from Theorem 3.1 by letting $t_{1}$ tends to zero.

By differentiating both sides of equation (3.16) with respect to $t_{1}, t_{2}$ and then setting $t_{1}=t_{2}=0$, we obtain the recurrence relations for product moments of gos from extended type II generalized logistic distribution in the form

$$
\begin{aligned}
& E\left[X^{i}(r, n, m, k) X^{j}(s, n, m, k)\right]=E\left[X^{j}(r, n, m, k) X^{i}(s-1, n, m, k)\right] \\
& \quad+\frac{j}{\alpha \gamma_{s}}\left\{E\left[X^{i}(r, n, m, k) X^{j-1}(s, n, m, k)\right]+E[\phi(X(r, n, m, k) X(s, n, m, k))]\right\}
\end{aligned}
$$

where

$$
\phi(x, y)=x^{i} y^{j-1} e^{-y} .
$$

Remark 3.1: Putting $m=0, k=1$ in (3.16) and (3.19), we obtain the recurrence relations for joint moment generating function and moments of order statistics for ex tended type II generalized logistic distribution as

$$
\begin{gathered}
\left(1-\frac{t_{2}}{\alpha(n-s+1)}\right) M_{X_{r, s: n}}^{(i, j)}\left(t_{1}, t_{2}\right)=M_{X_{r, s-1: n}}^{(i, j)}\left(t_{1}, t_{2}\right)+\frac{j}{\alpha(n-s+1)} M_{X_{r, s: n}}^{(i, j-1)}\left(t_{1}, t_{2}\right) \\
+\frac{1}{\alpha(n-s+1)}\left\{t_{2} M_{X_{r, s: n}}^{(i, j)}\left(t_{1}, t_{2}-1\right)+j M_{X_{r, s: n}}^{(i, j-1)}\left(t_{1}, t_{2}-1\right)\right\} \\
\text { Published by Atlantis Press } \\
\text { Copyright: the authors }
\end{gathered}
$$


and

$$
E\left[X_{r, s: n}^{(i, j)}\right]=E\left[X_{r, s-1: n}^{(i, j)}\right]+\frac{j}{\alpha(n-s+1)} E\left[X_{r, s: n}^{(i, j-1)}\right]+E\left[\phi\left(X_{r, s: n}\right)\right]
$$

Remark 3.2: Substituting $m=-1$ and $k \geq 1$, in (3.16) and (3. 19), we get recurrence relation for joint moment generating function and product moments of upper $k$ record values for extended type II generalized logistic.

\section{Acknowledgements}

The author acknowledge with thanks to Professor M. Ahsanullah, Department of Management Sciences, Rider University, Lawrenceville, NJ, USA, and the learned referee for helpful suggestions which resulted in improved presentation of the manuscript.

\section{References}

[1] Ahsanullah, M. and Raqab, M.Z. (1999): Recurrence relations for the moment generating functions of record values from Pareto and Gumble distributions. Stoch. Model. Appl., 2, 35-48.

[2] Al-Hussaini E.K., Ahmad, A.A. and Al-Kashif, M.A. (2005): Recurrence relations for moment and conditional moment generating functions of generalized order statistics. Metrika, 61, 199-220.

[3] Al-Hussaini E.K., Ahmad, A.A. and Al-Kashif, M.A. (2007): Recurrence relations for joint moment generating functions of generalized order statistics based on mixed population. J. Stat. Theory Appl., 6, 134-155.

[4] Balakrishnan N., Leung M. Y. (1988): Order statistics from the Type I generalized Logistic Distribution, Comm. Statist. Simulation and Computation. 17, 25-50.

[5] Balakrishnan, N. and Cohen, A.C. (1991): Order Statistics and Inference: Estimation Methods. Academic Press, San Diego.

[6] Kamps, U. (1995): A Concept of Generalized Order Statistics. B.G. Teubner Stuttgart.

[7] Kamps, U. and Cramer, E. (2001): On distribution of generalized order statis tics. Statistics, 35, 269-280.

[8] Kulshrestha A., Khan, R.U. and Kumar, D.(2013): On moment generating functions of generalized order statistics from Erlang-truncated exponential distribution. Open $J$. Statist., 2, 557-564.

[9] Kumar, D. (2012): Recurrence relations for marginal and joint moment generating functions of generalized logistic distribution based on lower $k$ record values and its characterization. ProbStats Fouram, 5, 47-53. 
[10] Kumar, D. (2013): Moment generating functions of lower generalized order statistics from generalized logistic distribution and its characterization. Pacific J. Appl. Math., $5,29-44$.

[11] Kumar, D. (2013): Relations for marginal and joint moment generating functions of Marshall-Olkin extended logistic distribution based on lower g eneralized order statistics and characterization. Amer. J. Math. Manag. Sci. 32, 19-39.

[12] Kumar, D. (2013): Relations for marginal and moments generating functions of extended type I generali zed logistic distribution based on lower generalized order statistics and characterization Tamsui Oxford journal of Mathematical Science, 29, 219-238.

[13] Pearson, K. (1968): Tables of Incomplete Beta Functions, 2nd ed. Cambridge, England: Cambridge University Press.

[14] Raqab, M.Z. and Ahsanullah, M. (20 00): Relations for marginal and jo int moment generating functions of record values from power function distribution. J. Appl. Statist. Sci., 10, 27-36.

[15] Raqab, M.Z. and Ahsanullah, M. (2003): On moment generating function of records from extreme value distribution. Pakistan J. Statist., 19, 1-13.

[16] Saran, J. and Pandey, A. (2003): Recurrence relations for marginal and joint moment generating functions of ge neralized order statistics from power function distribution. Metron, LXI, 27-33.

[17] Saran, J. and Pandey, A. (2012): Recurrence relations for marginal and joint moment generating functions of dual generalized order statistics fr om power function distribution. Pak. J. Statist. 28, 231-238.

[18] Shawky, A.I. and Bakoban, R.A. (2008): Characterization from exponentiated gamma distribution based on record values. J. Statist. Theory Appl., 7, 263-277. 